BNL-48784

Informal Report

\title{
EVALUATION OF PROPOSED SAMPLING AND ANALYTICAL METHODS FOR CARBONACEOUS HAZARDOUS AIR POLLUTANTS FOR THE CLEAN COAL TECHNOLOGY PROGRAM
}

\author{
M. A. Mazurek \\ Environmental Chemistry Division \\ Brookhaven National Laboratory \\ Upton, NY 11973 \\ and \\ L. M. Hildemann ${ }^{1}$ \\ Department of Civil Engineering \\ Stanford University \\ Stanford, CA $94305-4020$
}

March 1993

Prepared for
Office of Fossil Energy
U.S. Department of Energy

UNDER CONTRACT NO. DE-AC02-76CH00016 WITH THE UNITED STATES DEPARTMENT OF ENERGY. 


\section{INTRODUCTION}

To produce high quality technical data needed to assess the commercial readiness of advanced coal technologies, the U.S. Department of Energy Clean Coal Technology Program (DOE CCTP) must ensure that stack gas emissions are measured using the best proven methods of sampling and analysis. This is of special concem for the many toxic organic compounds which are regulated under Title III of the 1990 Clean Air Act Amendments (CAAA). The standard sampling and analytical methods which have been used without significant modification for the past 10 to 20 years (e.g. EPA Method 5) ${ }^{1,2}$ are not capable of determining quantitatively the extent to which these toxic organic compounds may be present in stationary source emissions. Hence, the need for robust sampling and analytical protocols that will meet current and future regulations is clear. The purpose of this report is to identify key issues relating to the sampling and analysis of carbonaceous hazardous air pollutants, and to discuss alternative methods that might be applied in a future air toxics program under consideration by the CCTP.

\section{LIMITATIONS OF SAMPLING METHODS}

Current standard methods, such as the EPA Method 5 train (EPA5) and the modified EPA Method 5 train (MEPA5) that have been proposed (e.g. Radian Field Chemical Emissions Monitoring Project, 1990 - REPA5) for stack sampling are based on a "hot gas" filtration approach. Although EPAS and its modifications are useful for obtaining data that are comparable to data collected previously, this protocol cannot measure accurately the quantity or partitioning of organic emissions present in the stack gases.

(1) Vapor phase and particle phase distribution

Distinguishing between the vapor and particulate phases of most of the Title III organic air pollutants in the stack emissions to account for differing fates in the environment will not be possible using the "hot gas" filtration approach used by EPAS and MEPAS.

The heated filter unit used by these methods to capture particulate matter significantly underestimates the amount of emitted material which will form aerosol once the stack emissions have been cooled and diluted to ambient conditions. In particular, semi-volatile organics are usually present in the gas phase of hot, undiluted stack emissions, but will condense onto existing particles once cooling and dilution occurs. Filtration of hot gases $\left(120 \pm 14^{\circ} \mathrm{C}\right)$ followed by cold-trapping the condensible gases simultaneously overestimates the amount of gas-phase organic emissions and underestimates the amount of organic particulate matter that is emitted. As an example, tests on a distillate oil-fired 
boiler found that EPA5 underestimated total emissions of fine organic particulate matter by approximately 10 -fold (cf. Figure 1$)^{4}$

Thus, the partitioning measured using hot filtration (EPA5, MEPA5) will not be representative of the ultimate partitioning (and hence, fate) of emissions in the atmosphere. In addition, discrepancies between the actual temperature of the stack emissions and the $120^{\circ} \mathrm{C}$ of the heated sampling probe will cause substantial test-to-test variations in the amount of condensate accumulating in the probe, and further confound attempts to compare the partitioning of emissions for different tests.

To obtain accurate information on the partitioning of emissions, a dilution stack sampling system must be used. This stack sampling approach uses a high degree of dilution to achieve temperatures and partial pressures that approximate the changes emissions undergo as a stack plume is dispersed downwind of a source. ${ }^{4}$ The system has a residence time that is long enough to allow supersaturated vapors to diffuse and subsequently condense onto existing particulate matter before the particulate-phase sample is collected.

(2) Particle size distribution

The size range of the bulk particulate matter sample collected by hot gas filtration is not appropriase for assessing environmental impacts of fine particles (e.g. respirable particles, visibility-reducing particles).

A particulate matter sample collected by combining a filter sample with the solvent rinses from a gooseneck nozzle and inlet line represents an approximation of the total suspended particles present in the stack gases. This sample spans the entire range of particle sizes present, including very coarse particles which will settle quickly out of the atmosphere and which neither penetrate deeply into the human respiratory tract nor impair visibility.

To evaluate the impact of particulate emissions, a well-defined size cut is needed for the particulate sample. Air quality criteria now consider particulate matter smaller than $10 \mu \mathrm{m}$ in diameter in setting primary standards. The fine fraction of particulate matter (less than $2 \mu \mathrm{m}$ in diameter) is viewed as posing the greatest environmental risk due to: (a) chemical composition; (b) persistence in the atmosphere (residence times on the order of weeks); and (c) aerodynamic capability to lodge deeply in human lungs. To collect a size-selective sample of particulate matter, a cyclone must be inserted upstream of the particle collection device(s) to remove particles larger than the size range of interest. ${ }^{4}$

(3) In-stack particle size distributions

Measurement of in-stack size distributions of the hot flue gases will not be 

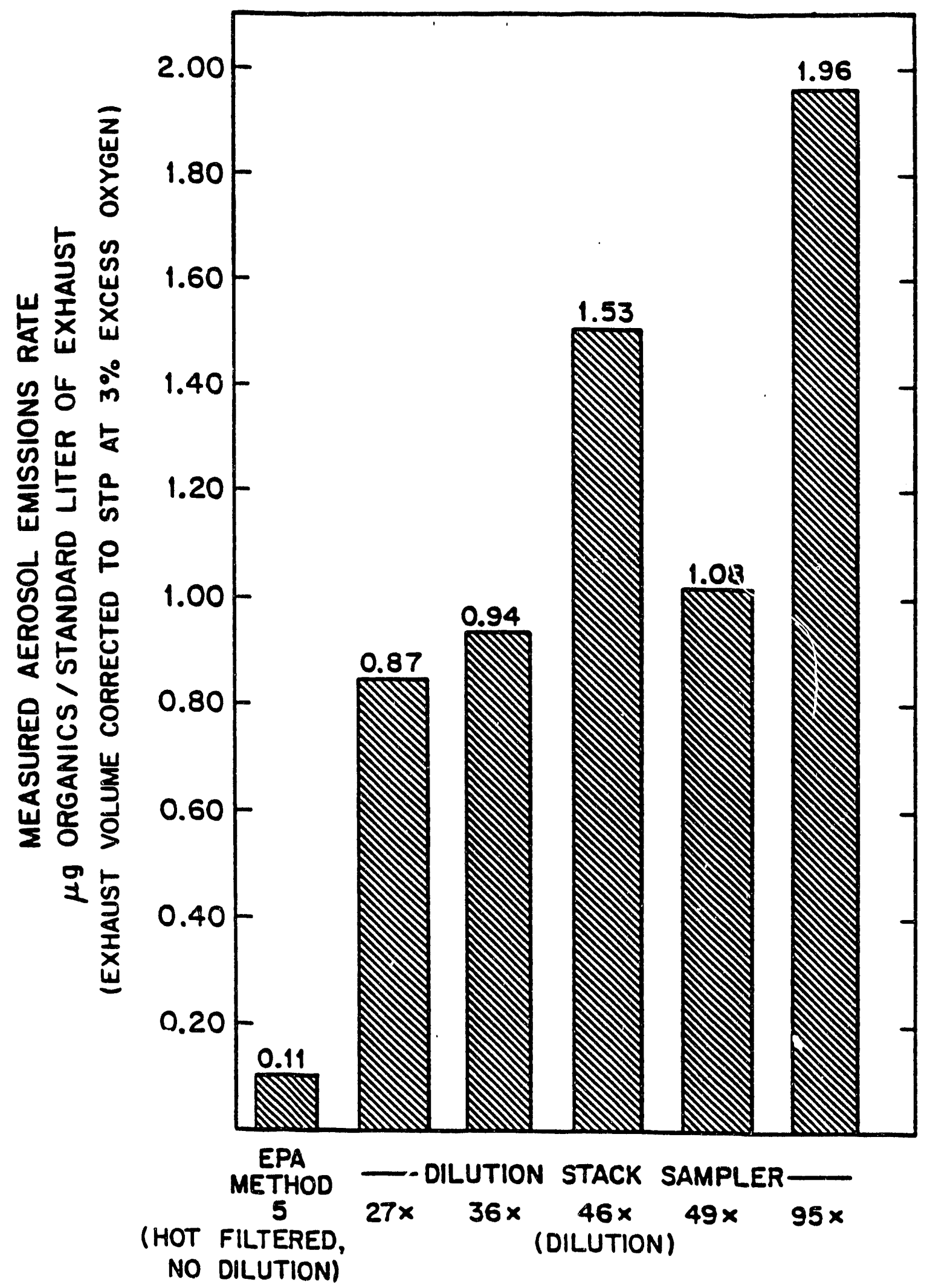

Figure 1. Organic carbon collected by filtration versus dilution sampling procedure for distillate oil-fired industrial boiler. ${ }^{4}$ 
representative of the ultimate size distribution of the emissions following cooling and dilution.

In undiluted stack gases, the size distribution evolves rapidly, so the measured distribution will be highly dependent on the temperature and time history of the emissions. Many nucleation processes (such as the formation of sulfuric acid mist) take place during the cooling and dilution that occurs after emission of the hot stack gases to the atmosphere. The fine particulate matter formed via such nucleation will not be collected by an impactor that samples the undiluted stack emissions. Hence, the in-stack size distribution measurements will not provide an accurate representation of the sizes of particulate matter actually contributed from the source to the atmosphere.

A dilution stack sampler is needed to obtain a more accurate representation of the actual particle size distribution of the emissions. ${ }^{4}$ The dilution process quenches the coagulation processes in a manner analogous to the normal atmospheric dispersion processes. More importantly, the cooling process causes some of the gas-phase components to become supersaturated, and these species proceed to nucleate and/or condense into the particulate phase. After allowing a sufficient residence time in the sampler for diffusion-limited condensation to occur, accurate size distribution measurements can be taken.

(4) Contamination by sampling equipment and procedural handling Components used in EPAS, MEPAS, and similar systems and the complicated handling procedures prescribed by these protocols will contribute significant organic contaminants to the sample.

The components of greatest concern present in the sampling trains modeled after EPA5 are: (a) silicone rubber gasket used to seal the heated filter holder; (b) Viton O-rings which seal other parts of the sampling train (e.g. glass probe liner); and (c) silicone grease used to seal the ground glass joints present in the impinger train. Each of these materials will off-gas organic compounds into the air stream during sampling. The concentration levels of these contaminants may be less than, comparable to, or much greater than concentrations of the individual molecular species that are actually present in the stack emissions. Under normal ambient conditions, ambient air sampling trains containing rubber components have collected detectable amounts of particulate silicone contaminants; these emissions of organic contaminants will increase as the temperature of the air stream increases.

At a minimum, the contaminants emitted by the sampling equipment will adversely affect the lower limits of detection achievable by gas chromatography (GC) and gas chromatography/mass spectrometry (GC/MS) procedures by 
increasing the background concentrations. It is likely that for some of the 189 hazardous air pollutants listed in Title III and/or some of the other semi-volatile organics of interest, the contaminants will coelute with these target species, causing difficulties in accurate identification and quantification. At worst, the

contaminant levels could cause certain of the target organic compounds to be completely unidentifiable by $\mathrm{GC}$ and $\mathrm{GC} / \mathrm{MS}$. In addition, high levels of organic contaminants present in the stack sampling equipment can be misidentified as emission components when in actuality these identified compounds are sampling artifacts.

To solve the problem of organic contamination of the samples by components of the sampler, the sampling system must not use any greases, oils, plastics, or rubber compounds at any point where the sample stream might come into contact with these materials.

Quality control procedures needed to evaluate sample modification and losses resulting from EPA5-type sampling protocols will be extremely difficult to implement and interpret. The sampling protocols prescribed by EPA5 and MEPA5 involve complicated presampling assembly of the system and detailed retrieval steps ior the samples thernselves. The multistep nature of these procedures, combined with the involvement of multiple operators in the sampling process, will cause substantial variations in sample quality, and will preclude identification of the cause(s) of sample contamination and loss.

To reduce sample loss and modification, a simplified collector is available which can be evaluated routinely for organic contaminants and sample loss." The stack sampling protocol must also specify collection of system blank samples on a test-by-test basis to account for the presence of background contaminants that may be incorporated into an actual stack emission sample. For accurate quality assurance, this system blank must be collected in a manner closely comparable to the way an actual sample is collected.

\section{LIMITATIONS OF ANALYTICAL METHODS}

Since the introduction of EPA5 20 years ago, significant advances have occurred in the analytical methods used for the identification and measurement of organic aerosol species from primary emission sources (e.g., ${ }^{6.7 .8}$ ). These technological improvements have not been incorporated into the updated version of EPA5 (e.g., ${ }^{2}$ ). Therefore, the analytical procedures specified in EPA5 cannot produce the quantitative data needed to accurately assess the presence of Title III carbonaceous hazardous air pollutants in stack emissions. 
Bulk organic chemical data

Use of the EPAS standard analytical protocol for organic compounds yields gravimetric information on the organics, but cannot provide data relating to the individual organic species present in the stack emissions.

Three types of samples are obtained from stack emission effluents by EPA5: (a) solid particles (collected on a pre-weighed glass fiber filter); (b) solvent-soluble matter (materials soluble in acetone that are rinsed from the sampler inlet line); and (c) water-soluble compounds (collected in the impingers). Gravimetric determinations are specified for the particulate matter and for the dried residue obtained from evaporation of the solvent rinse (accuracy of $\pm 0.1 \mathrm{mg}$ for both sample types). The quantity of water, only, is determined using gravimetric or volumetric methods for the impinger samples, and thus very polar organic constituents present in this aqueous sample are not measured. Clearly, no Title III organic compounds are identified by the gravimetric procedures described in EPA5 and no gravimetric data are produced which describe the fraction of carbonaceous matter that is present as solid particles.

Despite the lack of compound specific information that is obtained by EPA5, bulk chemical data concerning the total mass of carbonaceous material is important in the construction of mass balance profiles for stack emissions. As an example, differences in the bulk chemical composition of fine particle emissions have been determined recently for fine particle samples collected by a dilution stack sampling system (cf. Figure 2).9 Thus, a breakdown of the major chemical species (e.g. organic carbon, elemental carbon, sulfute, ammonium) present as solid particles in the hot stack emissions may be obtained from filters collected by EPAS and similar methods using current analytical protocols developed for stack emission tests. ${ }^{4}$

(2) Obtainable chemical information from stack emission samples

Chemical data obtained by REPAS do not reflect the physical state of the organic material measured, and thus will not aid in the design and implementation of control measures for Title III toxic organics present in stack emissions (i.e. control of particles versus vapors).

The recent REPAS protocol provides important modifications to the EPA5 "hot gas" filtration approach. ${ }^{3}$ Here, the types of samples collected (e.g. volatile organics by passivated cannister collection; semivolatile organics by sorbent trap collection; polar species by impinger collection; solid particles by in-stack filtration) permit individual organic compound identification that is otherwise not possible by the EPA5 protocol. However, the types of stack emission samples that are obtained using the Radian MEPA5 do not reflect the true distribution of organic matter that exists between the vapor phase and particle phase for reasons 


\section{SOURCE}

(a) Distillate Oil-Fired Boilers

(b) Catalyst-Equipped Automobiles

(c) Noncatalyst Automobiles

(d) Heavy-Duty Diesel Trucks

(e) Fireplace - Hard Wood

(f) Fireplace - Soft Wood

(g) Fireplace - Synthe:ic Log

(h) Meat Cooking Operations

(i) Natural Gas Home Appliances

(j) Cigarette Smoke

(k) Roofing Tar Pot Emissions

(l) Paved Road Dust

(m) Brake Dust

(n) Tire Dust

(o) Urban Vegetative Detritus
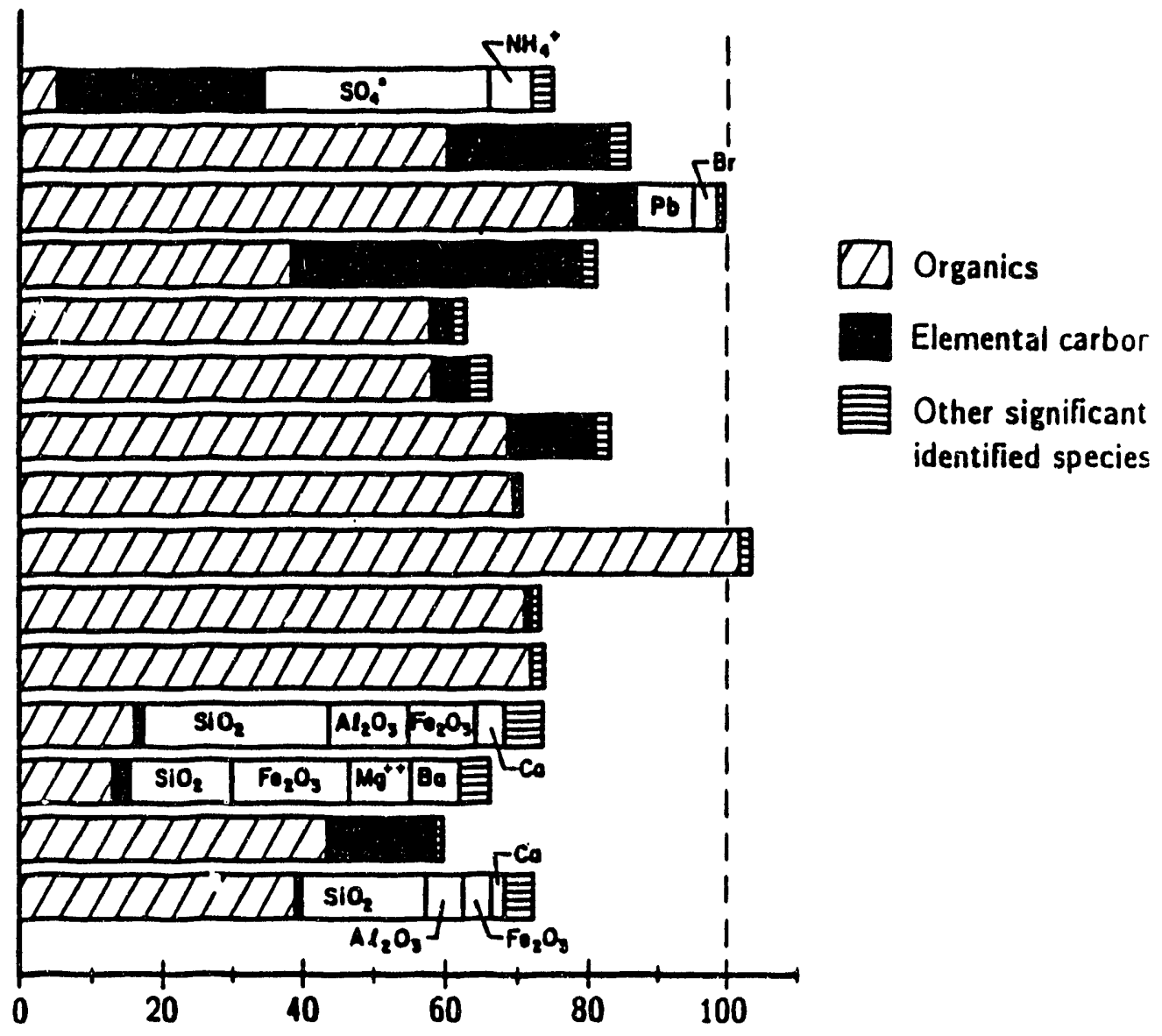

Percent of Total Fine Particulate Mass

Figure 2. Average chemical composition of fine particulate emissions from various source types. ${ }^{9}$ 
that have been discussed earlier. The Radian analytical protocol further obscures the distinction between vapor phase and particle phase organic compounds by compositing organic extracts from the sorbent trap, impinger condensate, and particle filter prior to analysis.

To preserve the chemical information that can be obtained for volatile and particle-bound organic species, composites of dissimilar sample types must be avoided.

(3) Measurement of individual organic compounds

Improvements in the analytical detection methods used for organic species characterization are incorporated into REPAS, but these methods are potentially applicable only to a small fraction of the Title III carbonaceous hazardous air pollutants.

The toxic organic chemicals identified in Title III comprise a wide range of molecular weight (volatility) and polarity, thus requiring multiple analytical methods to produce quantitative data. Volatile organics that are collected as a time-integrated sample directly from the stack $\left(120^{\circ} \mathrm{C}\right)$ are analyzed directly by GC and GC/MS. However, sample acquisition at $120^{\circ} \mathrm{C}$ will also collect higher molecular weight organic compounds that later will condense onto the sides of the stainless steel collection canister as the vapors cool to ambient temperatures. These compounds will not be measured accurately by the REPA5 protocol for volatile organic substances. The REPA5 protocol also includes analysis of semivolatile organic species and is based on GC and GC/MS analyses of the organic extracts obtained from the sampling train components. The method is adapted from a standard guideline established for the analysis of semivolatile organic compounds contained in solid waste. ${ }^{3,10}$ Many of the Title III toxic organic chemicals that would reasonably be present as aerosol in stack emissions from coal-fired power plants are very polar species (e.g. phenols, cresols, phthalates, glycols) that require specialized chromatographic conditions and derivatization steps in order to be detected by GC and GC/MS. These Title III compounds will not be measured accurately via REPA5. Therefore, a rigorous test of the overall REPAS analytical procedure is needed to establish its suitability for measurement of Title III organic compounds present in the stack emissions.

\section{CONCLUSIONS}

At present, no single stack sampling protocol (EPA5, MEPA5, REPA5, and dilution sampling methods) is adequate for evaluating completely stack gas emissions that will be tested as part of the CCTP. REPAS yields emissions data for organic compounds that are not representative of the physical or chemical composition of the aerosol. As an alternative method, 
dilution sampling of stack gases produces more accurate particle phase organic emissions data, but does not furnish information on volatile organics. A synthesis of the dilution sampling method and REPA5 sampling and analytical technologies will produce the high quality stack emissions data needed for future CCTP programs.

\section{REFERENCES}

1. Environmental Protection Agency (1971). Method 5 - Determination of particulate emissions from stationary sources. Federal Register 36(247):24888-24890.

2. Environmental Protection Agency (1991). Method 5 - Determination of particulate emissions from stationary sources. Federal Register 40(60):685-709.

3. Radian Corporation (1990). Generic sampling and analytical plan: Field chemical emissions monitoring project. Prepared for Electric Power Research Institute, Palo Alto, CA (EPRI Report RP3177-1).

4. Hildemann, L.M, Cass, G.R., and Markowski, G.R. (1989). A dilution stack sampler for collection of organic aerosol emissions: Design, characterization and field tests. Aerosol Sci. Technol. 10:193-204.

5. Mazurek, M.A., Cass, G.R., and Simoneit, B.R.T. (1991). Biological input to visibility reducing aerosol particles in the remote arid southwestern U.S. Environ. Sci. Technol. 25: 684-694.

6. Mazurek, M.A., Simoneit, B.R.T., Cass, G.R., and Gray, H.A. (1987). Quantitative high-resolution gas chromatography and high-resolution gas chromatography/mass spectrometry analyses of carbonaceous fine aerosol particles. Environ. Sci. Technol. 29:119-139.

7. Rogge, W.F., Hildemann, L.M., Mazurek, M.A., Cass, G.R., and Simoneit, B.R.T. (1991). Sources of fine organic aerosol. 1. Charbroilers and meat cooking operations. Environ. Sci. Technol. 25:1112-1125.

8. Hildemann, L.M., Mazurek, M.A., Cass, G.R., and Simoneit, B.R.T. (1991). Quantitative characterization of urban sources of organic aerosol by high-resolution gas chromatography. Environ. Sci. Technol. 25:1311-1325.

9. Hildemann, L.M., Markowski, G.R., and Cass, G.R. (1991). Chemical composition of emissions from urban sources of fine organic aerosol. Environ. Sci. Technol. 25:744-759. 
10. U.S. Environmental Protection Agency (1986). Test Methods for Evaluating Solid Waste - Physical/Chemical Methods. U.S. EPA, Washington, D.C., SW-846, third edition, revised. 

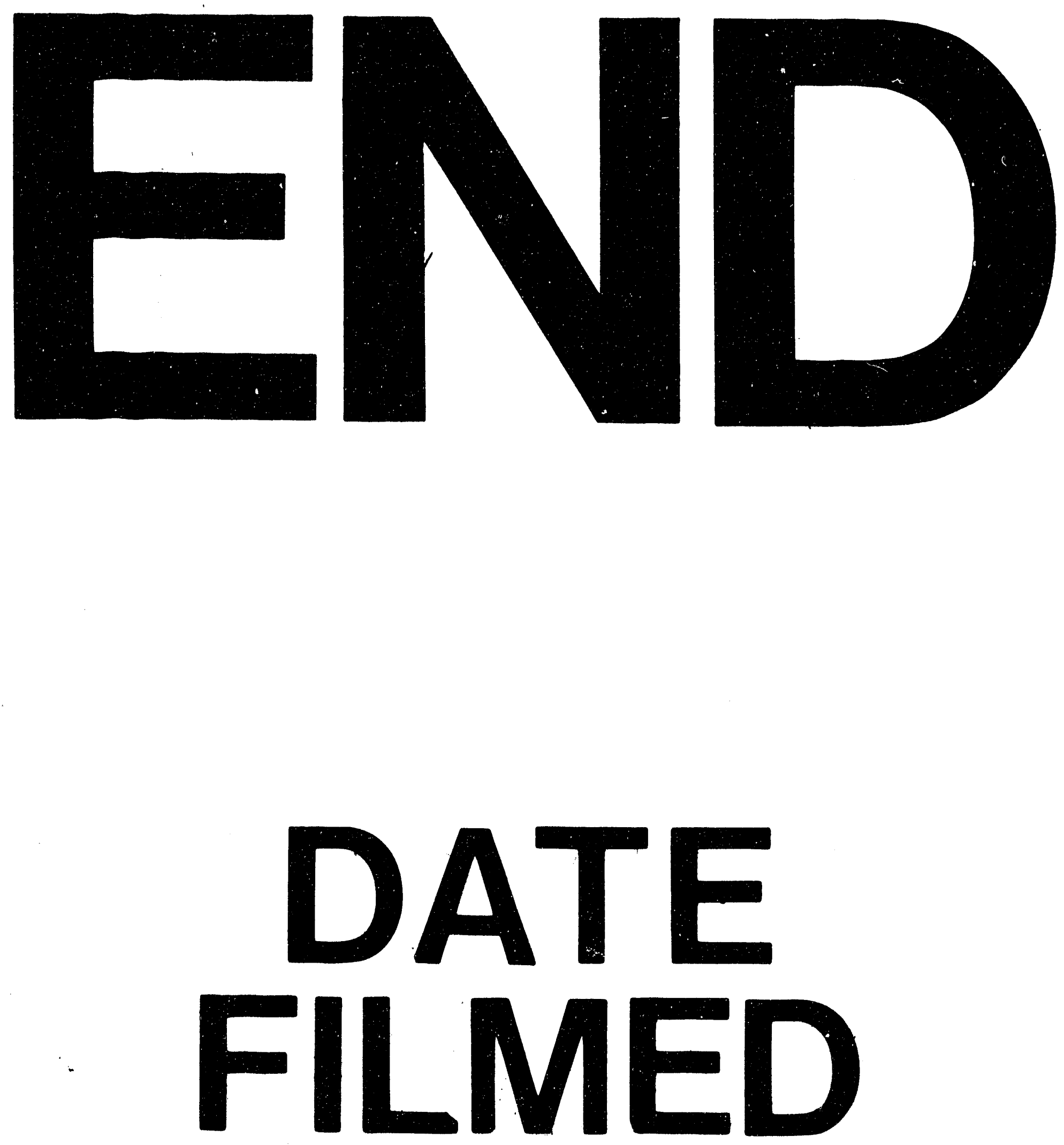

1

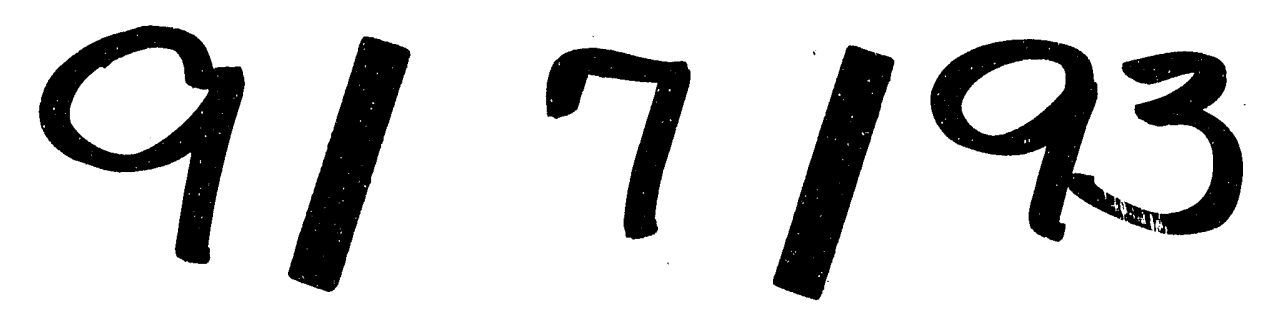

